\title{
Impact on liver position under breath-hold by computed tomography contrast agents in stereotactic body radiotherapy of liver cancer
}

\author{
Hideharu Miura $^{1,2}$, Shuichi Ozawa ${ }^{1,2}$, Minoru Nakao ${ }^{1,2}$, Yoshiko Doi ${ }^{1,2}$, Katsumaro Kubo ${ }^{1}$, \\ Masahiro Kenjo $^{1,2}$, Yasushi Nagata ${ }^{1,2}$ \\ ${ }^{1}$ Hiroshima High-Precision Radiotherapy Cancer Center, Hiroshima, Japan \\ ${ }^{2}$ Department of Radiation Oncology, Institute of Biomedical and Health Sciences, Hiroshima University, Hiroshima, Japan
}

\begin{abstract}
Background: We investigated variations in liver position relative to the vertebral bone for liver cancer treated with stereotactic body radiation therapy under expiratory phase breath-hold $(\mathrm{BH})$ for treatment with contrast-enhanced-computed tomography (CECT), non-CECT, and cone-beam computed tomography (CBCT).

Materials and methods: Seventeen consecutive patients using a contrast enhancement (CE) agent for the CT simulation session for this retrospective study were selected. The first computed tomography (CT) scan without the use of CE agent in the expiratory phase was used for treatment planning (pCT). The remaining three $\mathrm{CT}$ scans without a $\mathrm{CE}$ agent under expiratory phase $\mathrm{BH}$ were acquired successively without repositioning to evaluate the intra-fraction variation in liver position. Furthermore, a three-phase CT scan (arterial, portal, and late phases) accompanied by a CE agent under expiratory phase BH was acquired for target delineation. $\mathrm{CBCT}$ scans without the use of a $\mathrm{CE}$ agent under expiratory phase $\mathrm{BH}$ were acquired for treatment. Inter-fractional variations (non-CECT or CECT) in liver position were measured using the difference between CBCT and $\mathrm{pCT}$ or each 3 phase CECT images, respectively.

Results: The average \pm standard deviations for intrafractional, non-CECT interfractional variations, and CECT interfractional variations were $1.0 \pm 1.3,2.5 \pm 2.6$, and $6.4 \pm 6.4 \mathrm{~mm}$, respectively, in the craniocaudal (CC) direction. Intra- and inter-fractional variations in liver position were relatively small for non-CECT. However, significant inter-fractional liver position variations in CECT were observed in the expiratory phase BH. The position of the liver should be carefully considered when applying CECT images for image-guided radiotherapy.
\end{abstract}

Key words: image-guided radiotherapy; contrast enhancement agent; stereotactic body radiotherapy; liver cancer radiotherapy; intra-fraction liver position; inter-fraction liver position

Rep Pract Oncol Radiother 2021;26(6):1035-1044

\section{Introduction}

The success of stereotactic body radiation therapy (SBRT) requires precise localization and immobilization of targeted tumors to achieve accurate delivery during treatment. Respiration-induced organ motion constitutes a significant issue in radiation therapy. Moreover, while breathing, liver motion accounts for one of the most significant internal organ motions, ranging from 5 to $50 \mathrm{~mm}$

Address for correspondence: Hideharu Miura, Hiroshima High-Precision Radiotherapy Cancer Center, 3-2-2, Futabanosato, Higashi-ku Hiroshima, 732-0057, Japan, tel: +81-082-263-1330, fax: +81-082-263-1331; e-mail: miura@hiprac.jp

This article is available in open access under Creative Common Attribution-Non-Commercial-No Derivatives 4.0 International (CC BY-NC-ND 4.0) license, allowing to download articles and share them with others as long as they credit the authors and the publisher, but without permission to change them in any way or use them commercially 
$[1,2]$. Many proposed techniques consider respiration-induced organ motion, including abdominal compression, respiratory gating, real-time tumor tracking, expiratory breath-hold $(\mathrm{BH})$, deep inspiration $\mathrm{BH}(\mathrm{DIBH})$, and $\mathrm{BH}$ with an active breathing coordinator $(\mathrm{ABC})$ [3-17]. The respiration-induced organ motion management technique affects the intra- and inter-fraction planning margins. The $\mathrm{BH}$ technique with an external surrogate is a non-invasive and cost-effective method for the delivery of high-dose radiation to tumors while reducing the dose to the healthy liver. Inter-BH reproducibility in the end-expiratory phase is more stable than that in the end-inspiratory phase [4-6]. Additionally, the best correlation between the motion of an external surface and internal fiducial markers for the liver in respiratory-based computed tomography $(\mathrm{CT})$ is observed during the expiratory phase [7]. Therefore, we used the Abches system (Apex Medical, Tokyo, Japan) with $\mathrm{BH}$ in the expiratory phase [18-20].

Kilo-voltage cone-beam CT (kV-CBCT) is an essential tool for patient positioning and target verification. Using $\mathrm{kV}-\mathrm{CBCT}$ scans, lung tumors can be directly registered with a planning CT (pCT) scan. However, liver tumors are not visible on CBCT images without using contrast enhancement (CE) agents, and the setup relies on surrogates, such as an implanted fiducial marker $[7,11]$, a diaphragm dome $[8,12]$, or the liver organ shape itself $[9,13]$. Image guidance using fiducial markers implanted around tumors help to achieve a more accurate patient setup while considering organ motion [7]. For cases without fiducial markers, the diaphragm is often used as a typical surrogate for liver tumors. A good correlation between liver tumor motion and diaphragm motion exists in the craniocaudal (CC) and anteroposterior (AP) directions but not in the left-right (LR) direction [8].

CT datasets without a CE (non-CECT) agent are usually employed for treatment planning. In addition to non-CECT, CT with a CE agent (CECT) obtained under $\mathrm{BH}$ in the radiation therapy treatment position is used for target delineation. The target tumor is contoured using CT imaging or magnetic resonance imaging (MRI) with a CE agent because liver tumors cannot be visualized without a $\mathrm{CE}$ agent [21]. Some studies have reported the use of CECT for liver SBRT in treatment planning or image-guided radiotherapy (IGRT) [9-17]. However, a CE agent may cause side effects, such as elevated heartbeat [22], and could result in patient's anxiety. As a result, the patient may not keep the same timing of each breath-hold. The position of the liver in the pCT while using a $\mathrm{CE}$ agent and the position of the liver in the daily CBCT may be significantly different. The position of the liver may lead to deformation. The position of the liver is prone to deformation due to respiratory motion, resulting in variations in the shape of the liver between different scans. Furthermore, inter-fraction liver position in breath-hold CT images might occur using an inappropriate phase CT image owing to different $\mathrm{BH}$ timing on each CECT acquisition.

The purpose of this study was to investigate the reproducibility of liver position in CT images with and without the use of a $\mathrm{CE}$ agent relative to the vertebral bone on CBCT. We also investigated the intra-fractional variation in liver position.

\section{Materials and methods}

\section{Patients}

Between November 2018 and November 2019, 17 consecutive patients using a CE agent for the CT simulation session for this retrospective study were selected. Patient characteristics are summarized in Table 1 . This study was approved by the Institutional Review Board of the Hiroshima University (E-947). The Abches system was used to monitor to self-control respiratory motion and tumor displacement during the simulation and treatment sessions [18-20]. The Abches system involves thoracic and abdominal contacts and a respiratory level indicator panel with a pointer. This pointer moves along with the vertical motion of the two contacts on the chest and abdominal wall during respiration. The panel has three markers that can be freely placed at arbitrarily selected positions. These markers indicate the position of the $\mathrm{BH}$ for the pointer. The real-time position of the indicator can be monitored via a video camera system attached to the Abches system. Patients were placed in a supine position using a radiography simulator (VersiFlex VISTA, Hitachi Medical Co., Kashiwa, Japan). The patients practiced holding their breath during the expiratory phase. Simultaneously, a radiological technologist checked both positions of the Abches pointer using a video monitor and the diaphragm using $\mathrm{X}$-ray fluoroscopy in the control room. The $\mathrm{BH}$ ma- 
Table 1. Patient characteristics

\begin{tabular}{|c|c|c|c|c|c|}
\hline No. & Age (y) & Gender & TNM stage & location & CTV [cc] \\
\hline 1 & 74 & M & T1NOMO & S8 & 0.9 \\
\hline 2 & 70 & M & T1N0M0 & S2 & 1.7 \\
\hline 3 & 70 & M & T2NOMO & S5 & 3.0 \\
\hline 4 & 74 & M & T1N0M0 & S5 & 4.8 \\
\hline 5 & 65 & M & T2NOMO & S5 & 2.8 \\
\hline 6 & 70 & M & T2NOMO & S8 & 4.1 \\
\hline 7 & 51 & $\mathrm{~F}$ & T1NOMO & S1 & 27.7 \\
\hline 8 & 74 & M & T2NOMO & S1 & 1.4 \\
\hline 9 & 66 & M & T2NOMO & S5 & 2.4 \\
\hline 10 & 85 & $M$ & T1NOMO & S8 & 5.2 \\
\hline 11 & 74 & $M$ & T1N0M0 & S8 & 6.3 \\
\hline 12 & 80 & M & T1NOMO & S8 & 26.3 \\
\hline 13 & 73 & M & T2NOMO & S5 & 2.6 \\
\hline 14 & 73 & $\mathrm{~F}$ & T1N0M0 & S7 & 0.7 \\
\hline 15 & 66 & $M$ & T1N0M0 & S4 & 7.3 \\
\hline 16 & 80 & $M$ & T2NOMO & S6 & 5.5 \\
\hline 17 & 85 & M & T1NOMO & S6 & 1.3 \\
\hline
\end{tabular}

$\mathrm{M}$ - male; $\mathrm{F}$ - female; $\mathrm{S}$ - segment; $\mathrm{T}$ - size or direct extent of the primary tumor, $\mathrm{N}$ - degree of spread to regional lymph nodes, $\mathrm{M}$ - presence of distant metastasis, S - segment; CTV — clinical target volume

neuver was then performed three times, with each $\mathrm{BH}$ maintained for as long as possible. The length of the $\mathrm{BH}$ was dependent on the patient's comfort level. Using X-ray fluoroscopy, the reproducibility of the diaphragm position was confirmed to be within $5 \mathrm{~mm}$ for each respiratory phase.

\section{CT simulation}

All patients underwent CT scans (Optima CT 580 W; GE Healthcare, Milwaukee, WI, USA) in a supine position with their arms above their head in either a chest board or an evacuated immobilization bag with a leg immobilizer placed under their knees. A total number of 7 CT scans per patient were acquired at the expiratory phase. A CT scan without a $\mathrm{CE}$ agent under expiratory phase $\mathrm{BH}$ was used for treatment planning. Three verification CT scans were successively acquired without repositioning to investigate inter- $\mathrm{BH}$ reproducibility. Once each $\mathrm{BH}$ was completed, a sufficient free-breathing period was provided to prepare for the next $\mathrm{BH}$. These CT scans were acquired without the use of a CE agent. The reproducibility of expiratory inter- $\mathrm{BH}$ confirmed the intra-fractional variation in the liver position. The three $\mathrm{CT}$ phases (arterial, portal, and late) at the expiratory $\mathrm{BH}$ were scanned to contour the target at 30,60 , and $120 \mathrm{~s}$ after the intravenous administration of a CE agent (Iopamiron 370, Bayer Schering Pharma, Osaka, Japan). These three phase CT scans with a CE agent were labeled as CECT1, CECT2, and CECT3, respectively. The reproducibility of the expiratory inter-BH accompanied by the $\mathrm{CE}$ agent confirmed the inter-fraction variation in liver position that would occur if these CECT image was used for IGRT instead of a plain CT image. The following imaging parameters were used for treatment planning: CT, $120 \mathrm{kV}, 400 \mathrm{~mA}$; matrix resolution, $512 \times 512$; pixel size, $0.98 \mathrm{~mm}$ in the LR and AP directions; and $1.25 \mathrm{~mm}$ in the CC direction.

\section{Treatment plan}

The CT images were transferred to a RayStation treatment planning system (RaySearch Medical Laboratories AB, Stockholm, Sweden), and the treatment plan CT images were fused on CECT images or MRI to delineate a target for all patients. The magnetic resonance apparatus was a 3.0 Tesla system (Discovery MR750w; GE Healthcare, Milwaukee, WI, USA). The patients were positioned using the same immobilization device that was used in the CT simulation and 
they received a dose of $0.1 \mathrm{~mL} / \mathrm{kg}$ Gd-EOB-DTPA (Primovist: Bayer Schering Pharma, Berlin, Germany) injected at $1.0 \mathrm{~mL} / \mathrm{s}$. The hepatobiliary phase was acquired $20 \mathrm{~min}$ after the intravenous administration of an MRI CE agent. A radiation oncologist then delineated the gross tumor volume (GTV) and liver; no additional margin was added to define the clinical target volume (CTV). The planning target volume (PTV) was created by expanding the CTV by $5-8 \mathrm{~mm}$ in all directions to account for $\mathrm{BH}$ uncertainty, setup uncertainty, and mechanical inaccuracy. The isocenter was placed at the center of the PTV. Volumetric modulated arc therapy with a beam energy of 10 MV in flattening filter-free mode was used for all patients. In accordance with local protocols, the patients in this study were prescribed a dose of 40 Gy in four fractions which was administered to $95 \%$ of the PTV. The dose was inhomogeneously prescribed to the PTV with a near-maximum dose $\left(D_{2 \%}\right)$ and it ranged from $125 \%$ to $130 \%$. The dose constraints of the organ at risk (OAR) included the liver, stomach, esophagus, duodenum, large bowel, small bowel, gall bladder, spinal cord, and skin under our clinical protocol.

\section{Treatment session}

During the treatment session, the patients were positioned using the same immobilization device used in the simulation and then aligned with infrared markers equipped in the ExacTrac system (Brainlab, Feldkirchen, Germany). Stereoscopic images were used to perform a bony anatomy match on the vertebral bone, and they were corrected for setup errors in six dimensions using an Exact Couch (Brainlab, Feldkirchen, Germany). Following setup correction, a $\mathrm{kV}-\mathrm{CBCT}$ scan without a $\mathrm{CE}$ agent under expiratory phase $\mathrm{BH}$ was acquired using an on-board imaging system equipped with a TrueBeamSTx (Varian Medical Systems, Palo Alto, CA) linear accelerator. The CBCT acquisition required $35 \mathrm{~s}$ for a $200^{\circ}$ rotation; consequently, three to four BHs were necessary to acquire the $\mathrm{CBCT}$ scan. Image registration was performed based on the soft-tissue alignment of the liver by registering $\mathrm{CBCT}$ with $\mathrm{pCT}$.

\section{Analysis}

Figure 1 shows a schematic of the imaging acquisition and organ motion definition. The translations (LR, AP, and CC directions) and rotations

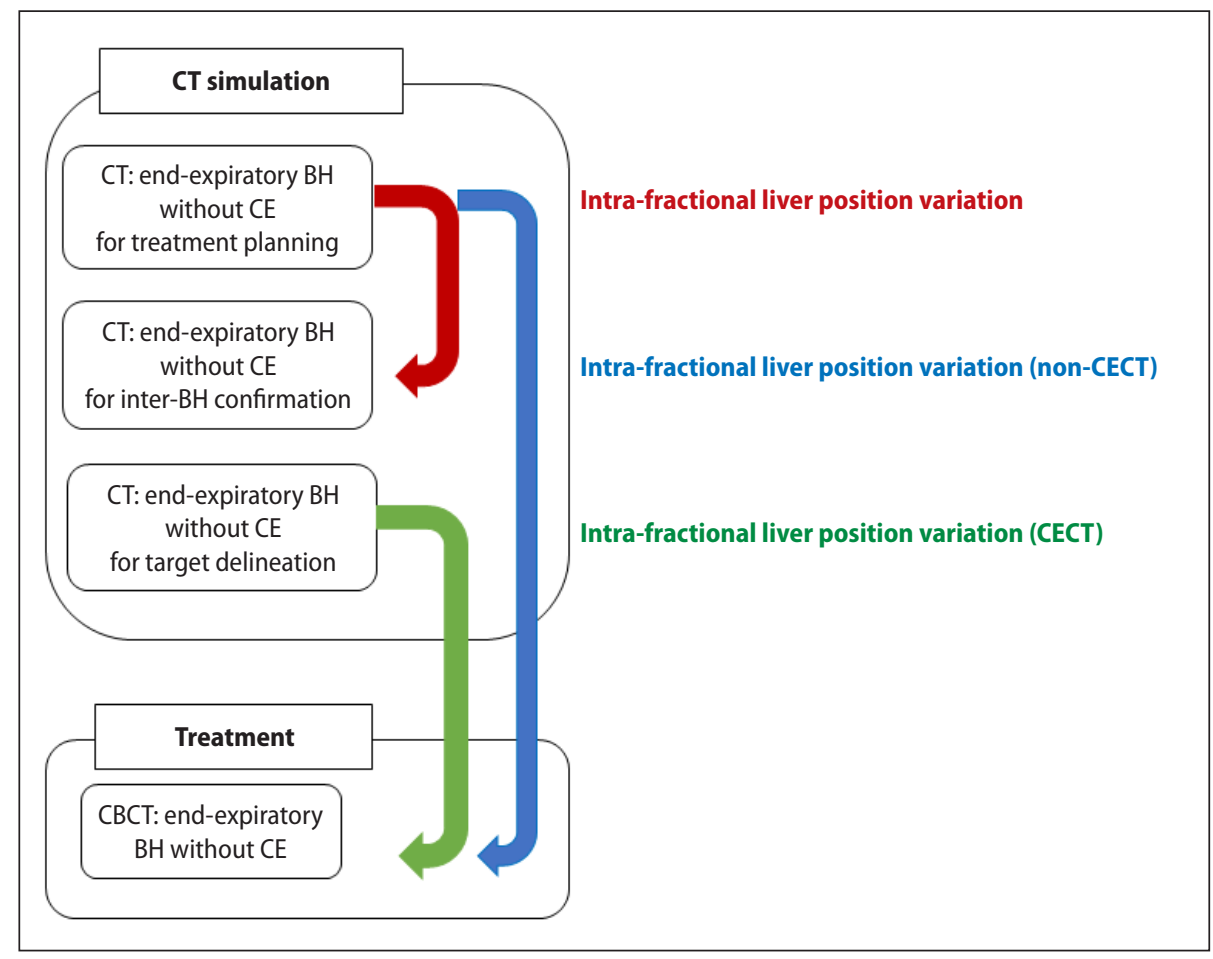

Figure 1. Schematic of image acquisition and organ motion definition; $\mathrm{BH}$ - breath holt; $\mathrm{CE}$ - contrast enhancement; CECT - contrast-enhanced-computed tomography; CBCT - cone-beam computed tomography 
(pitch around LR, roll around CC, and yaw around AP) were measured to evaluate the intra- and inter-fractional variations in liver position using RayStation TPS. Rigid image registration to the vertebral bone was performed to remove potential residual patient position errors. Three repeated CT images without use of a $\mathrm{CE}$ agent were used to investigate the intra-fractional variation in liver position. The CBCT images were transferred to the RayStation TPS. Position difference in liver position between non-CECTs and CBCT was defined as inter-fractional (non-CECT) variation in liver position. Position difference in liver position between each three phase CT with $\mathrm{CE}$ agent and $\mathrm{CBCT}$ was defined as inter-fractional (CECT) variation in liver position. Detecting GTV on CT and $\mathrm{CBCT}$ images without using $\mathrm{CE}$ agents is typically challenging owing to the low image contrast.
Image registrations were performed using $\mathrm{CBCT}$ images with pCT or each of the 3 phase CT images based on soft-tissue alignment of the liver contour and vessel information.

The data were analyzed using Wilcoxon signed-rank tests with the statistical significance set at $\mathrm{p}<0.05$, applying R version 3.5.2 (www.r-project.org).

\section{Results}

The distributions of translation and rotation intra- and inter-fractional (non-CECT and CECT) variations in liver position relative to the vertebral bone using the Abches system are shown in Figure 2. The translation and rotation intra- and inter-fractional (non-CECT and CECT) variations in liver position relative to the vertebral bone are summarized in Table 2.
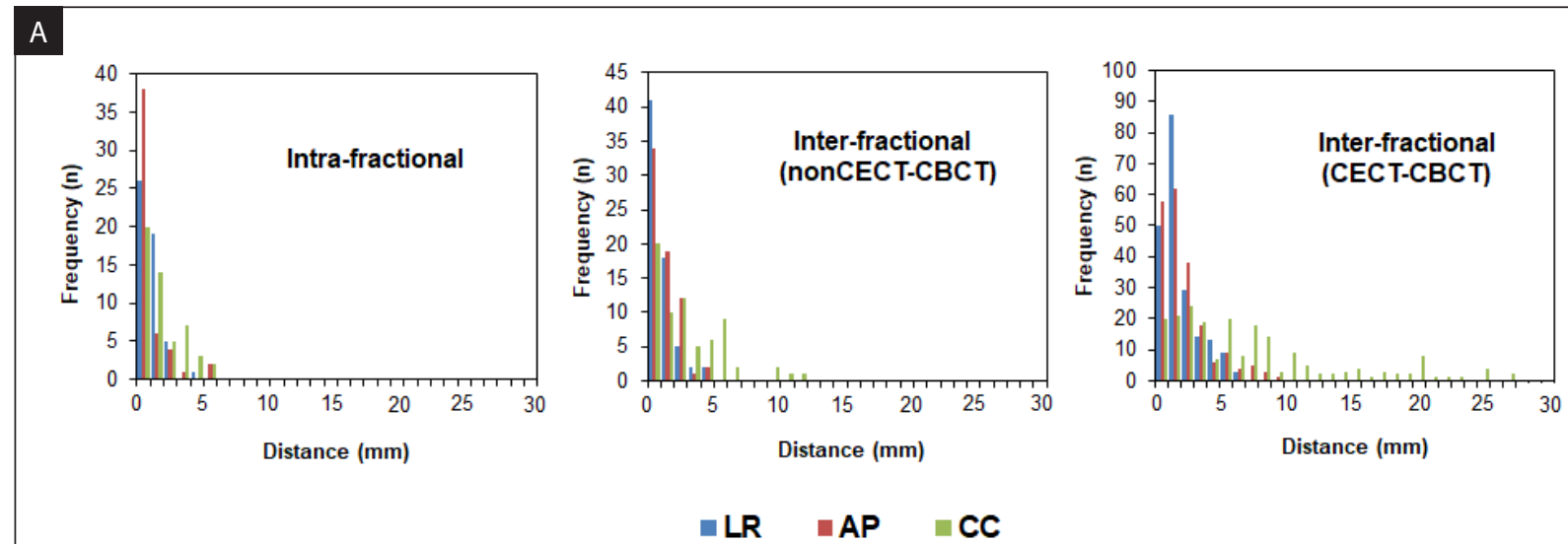

B
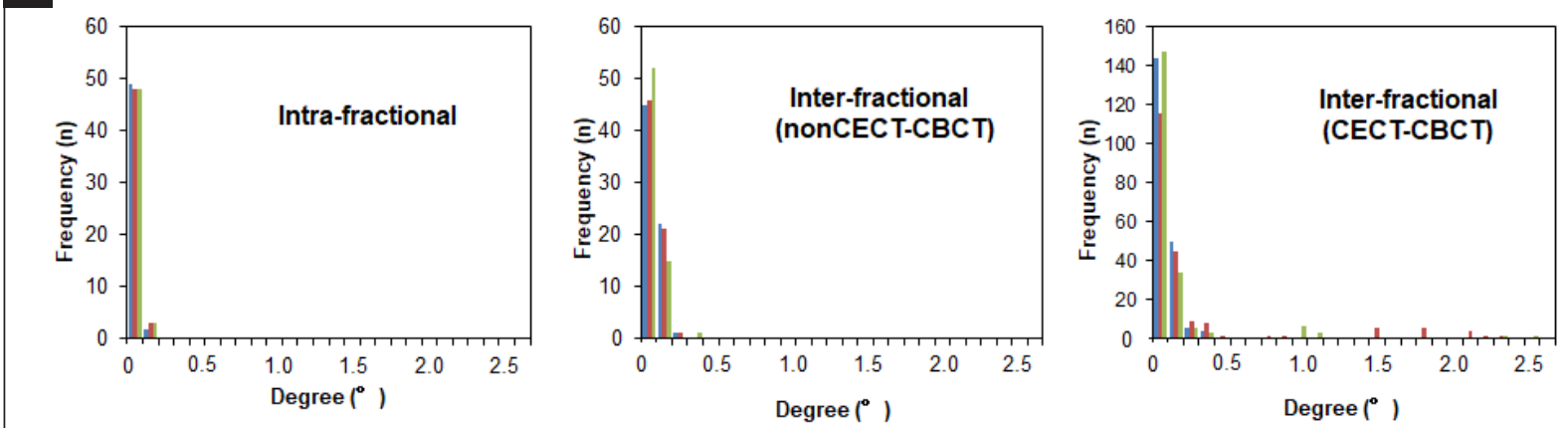

- Pitch $\quad$ Roll $\square$ Yaw

Figure 2. A. Translation of intra- and inter-fractional variations (non-CECT or CECT) in liver position in the left-right (LR), anteroposterior (AP), and craniocaudal (CC) directions, respectively; B. Rotation of intra- and inter-fractional variations (non-CECT or CECT) in liver position around LR (pitch), CC, (roll), and AP (yaw) axis, respectively. CECT - contrast-enhanced-computed tomography; CBCT — cone-beam computed tomography 
Table 2. Average and standard deviation of the translation and rotation liver positions relative to the vertebral bone on planning computed tomography (CT)

\begin{tabular}{|c|c|c|c|c|c|c|c|}
\hline & & LR [mm] & $\mathrm{AP}[\mathrm{mm}]$ & $\mathrm{CC}[\mathrm{mm}]$ & Pitch $\left({ }^{\circ}\right)$ & Roll $\left({ }^{\circ}\right)$ & Yaw $\left({ }^{\circ}\right)$ \\
\hline Intra-fraction & $\mathrm{n}=51$ & $\begin{array}{l}0.4 \pm 0.6 \\
(0.0-3.4)\end{array}$ & $\begin{array}{l}0.4 \pm 0.9 \\
(0.0-4.3)\end{array}$ & $\begin{array}{l}1.0 \pm 1.3 \\
(0.0-5.0)\end{array}$ & $\begin{array}{l}0.0 \pm 0.0 \\
(0.0-0.1)\end{array}$ & $\begin{array}{l}0.0 \pm 0.0 \\
(0.0-0.1)\end{array}$ & $\begin{array}{l}0.0 \pm 0.0 \\
(0.0-0.1)\end{array}$ \\
\hline $\begin{array}{l}\text { Inter-fraction } \\
(\mathrm{pCT}-\mathrm{CBCT})\end{array}$ & $n=68$ & $\begin{array}{l}0.6 \pm 1.0 \\
(0.0-4.0)\end{array}$ & $\begin{array}{l}0.8 \pm 1.0 \\
(0.0-4.0)\end{array}$ & $\begin{array}{c}2.5 \pm 2.6 \\
(0.0-11.0)\end{array}$ & $\begin{array}{l}0.0 \pm 0.1 \\
(0.0-0.2)\end{array}$ & $\begin{array}{l}0.0 \pm 0.1 \\
(0.0-0.2)\end{array}$ & $\begin{array}{l}0.0 \pm 0.1 \\
(0.0-0.3)\end{array}$ \\
\hline $\begin{array}{l}\text { Inter-fraction } \\
\text { (CECT1-CBCT) }\end{array}$ & $n=68$ & $\begin{array}{l}1.0 \pm 1.2 \\
(0.0-5.0)\end{array}$ & $\begin{array}{l}1.3 \pm 1.6 \\
(0.0-6.8)\end{array}$ & $\begin{array}{c}5.3 \pm 4.7 \\
(0.0-19.8)\end{array}$ & $\begin{array}{l}0.0 \pm 0.1 \\
(0.0-0.2)\end{array}$ & $\begin{array}{l}0.2 \pm 0.4 \\
(0.0-1.7)\end{array}$ & $\begin{array}{l}0.1 \pm 0.2 \\
(0.0-0.9)\end{array}$ \\
\hline $\begin{array}{l}\text { Inter-fraction } \\
\text { (CECT2-CBCT) }\end{array}$ & $n=68$ & $\begin{array}{l}1.1 \pm 1.3 \\
(0.0-4.4)\end{array}$ & $\begin{array}{l}1.6 \pm 2.0 \\
(0.0-8.8)\end{array}$ & $\begin{array}{c}6.6 \pm 7.0 \\
(0.0-27.7)\end{array}$ & $\begin{array}{l}0.0 \pm 0.1 \\
(0.0-0.3)\end{array}$ & $\begin{array}{l}0.3 \pm 0.6 \\
(0.0-2.2)\end{array}$ & $\begin{array}{l}0.2 \pm 0.5 \\
(0.0-2.3)\end{array}$ \\
\hline $\begin{array}{l}\text { Inter-fraction } \\
\text { (CECT3-CBCT) }\end{array}$ & $\mathrm{n}=68$ & $\begin{array}{l}1.4 \pm 1.5 \\
(0.0-5.1)\end{array}$ & $\begin{array}{l}1.6 \pm 2.0 \\
(0.0-7.6)\end{array}$ & $\begin{array}{c}7.3 \pm 7.2 \\
(0.0-25.6)\end{array}$ & $\begin{array}{l}0.0 \pm 0.0 \\
(0.0-0.2)\end{array}$ & $\begin{array}{l}0.2 \pm 0.4 \\
(0.0-1.7)\end{array}$ & $\begin{array}{l}0.1 \pm 0.2 \\
(0.0-0.9)\end{array}$ \\
\hline $\begin{array}{l}\text { Inter-fraction } \\
\text { (All CECT-CBCT) }\end{array}$ & $\mathrm{n}=204$ & $\begin{array}{l}1.2 \pm 1.3 \\
(0.0-5.1)\end{array}$ & $\begin{array}{l}1.5 \pm 1.9 \\
(0.0-8.8)\end{array}$ & $\begin{array}{c}6.4 \pm 6.4 \\
(0.0-27.7)\end{array}$ & $\begin{array}{l}0.0 \pm 0.1 \\
(0.0-0.3)\end{array}$ & $\begin{array}{l}0.2 \pm 0.5 \\
(0.0-2.2)\end{array}$ & $\begin{array}{l}0.1 \pm 0.4 \\
(0.0-2.3)\end{array}$ \\
\hline
\end{tabular}

The ranges are shown in parentheses. LR — left-right; AP — anteroposterior; CC — craniocaudal. The rotational angles in pitch, roll, and yaw, with corresponding rotational axes in the LR, CC, and AP directions, respectively. CECT1, CECT2, and CECT3 indicates arterial, portal, and late phase CTs, respectively

\section{Intra-fractional variation in liver position}

The average and standard deviation of intra-fractional variation in liver position were $0.4 \pm 0.6$, $0.4 \pm 0.9$, and $1.0 \pm 1.3 \mathrm{~mm}$ in the LR, AP, and CC directions, respectively. Three patients exhibited an intra-fractional variation of $>3 \mathrm{~mm}$ in the liver position in the CC direction, and no displacement greater than $5 \mathrm{~mm}$ was observed in any direction in these patients. The liver rotation for repeated CT scans without the use of a CE agent was below $0.1^{\circ}$ in all rotations.

\section{Inter-fractional (non-CECT) variation in liver position}

The average and standard deviation of inter-fractional (non-CECT) variation in liver position were $0.6 \pm 1.0,0.8 \pm 1.0$, and $2.5 \pm 2.6 \mathrm{~mm}$ in the LR, AP, and CC directions, respectively. Seven patients ex- hibited interfractional variations greater than $5 \mathrm{~mm}$ in the liver. These patients had an inter-fractional variation in liver position greater than $5 \mathrm{~mm}$ in the CC direction only. Among them, six patients had inter-fractional variation in liver position between 5 and $10 \mathrm{~mm}$, and one had an inter-fractional variation in liver position greater than $10 \mathrm{~mm}$. The liver rotation for $\mathrm{CBCT}$ without the use of a $\mathrm{CE}$ agent was below $0.3^{\circ}$ in all rotations.

\section{Inter-fractional (CECT) variation in liver position}

Figure 3 depicts the patients with poor $\mathrm{BH}$ relative to the vertebral bone during CECT. The average and standard deviation of inter-fractional (CECT) variation in liver position were $1.2 \pm 1.3,1.4 \pm 1.7$, and $6.4 \pm 6.4 \mathrm{~mm}$ in the LR, AP, and CC directions, respectively. The average and standard deviations

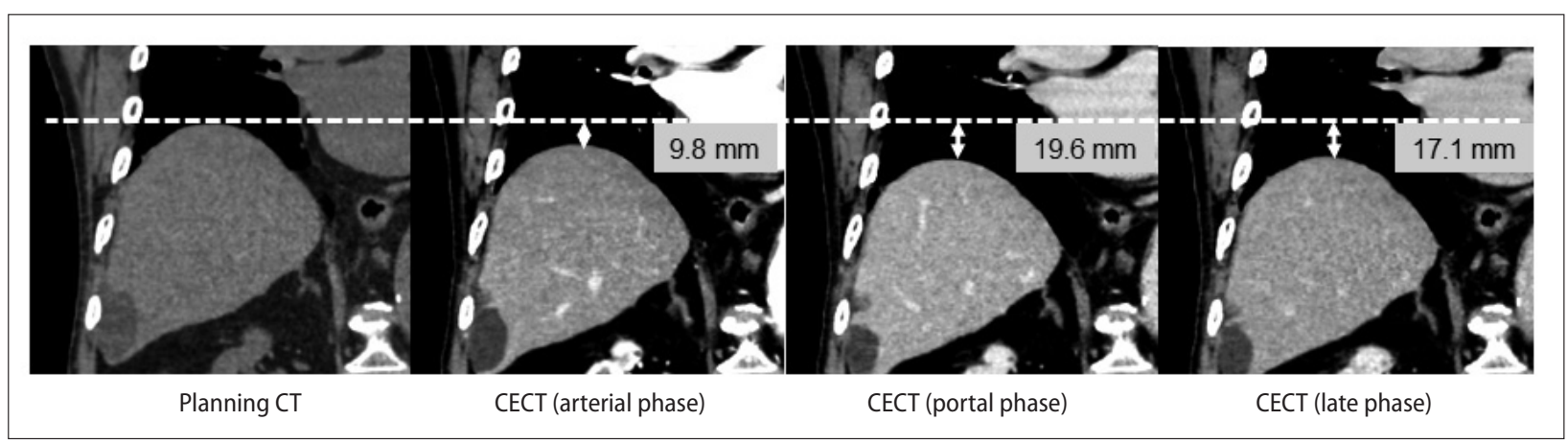

Figure 3. Three sets of contrast-enhanced-computed tomographies (CECTs) were registered using rigid image registration based on the vertebral bone. The displacements on the arterial, portal, and late phases of the liver for this patient with the most significant shift were 15.2, 22.7, and $20.6 \mathrm{~mm}$ in the CC direction. It should be noted that this figure shows the displacements of the liver edges between planning CT at the selected CT particular slice 
for inter-fractional (CECT1, CECT2 and CECT3) variations in liver position were $5.3 \pm 4.7,6.6 \pm 7.0$, and $7.3 \pm 7.2 \mathrm{~mm}$, respectively, in the CC direction.

Thirteen patients had inter-fractional (CECT) variation in liver position greater than $5 \mathrm{~mm}$ in any direction. Among them, twelve patients had inter-fractional (CECT) variation in liver position between 5 and $10 \mathrm{~mm}$, and five patient had inter-fractional (CECT) variation in liver position greater than $10 \mathrm{~mm}$. The maximum variation in liver position was $27.7 \mathrm{~mm}$ in the CC direction. The most significant internal rotations for $\mathrm{CT}$ using a $\mathrm{CE}$ agent occurred around the AP axis (yaw), with a maximum of $2.3^{\circ}$.

\section{Comparison between the non-CECT and CECT scans}

For the LR and AP direction, the inter-fractional variations in liver position showed no statistically significant differences between the non-CECT and CECT scans, with 0.347 and 0.080 , respectively. Compared to the non-CECT, the inter-fractional (CECT) variation in liver position on average increased $3.9 \mathrm{~mm}(\mathrm{p}<0.001)$.

\section{Discussion}

\section{Intra-fractional variation in liver position}

First, we investigated the intra-fractional variation in liver position under the expiratory phase $\mathrm{BH}$ with the Abches system using non-CECT. The average intra-fractional variation in liver position in the CC direction under non-CECT was $1.0 \mathrm{~mm}$, similar to that of the intra-fractional variation in liver position in previous studies [5-17]. The internal rotation of the liver is very unlikely to be detected on repeated CT scans without the use of CE. According to Lu et al. [9], the mean absolute intra-fractional displacements for $\mathrm{BH}$ with $\mathrm{ABC}$ in the liver in the LR, AP, and CC directions are $0.59 \mathrm{~mm}, 1.16 \mathrm{~mm}$, and $1.33 \mathrm{~mm}$, with standard deviations of $0.75 \mathrm{~mm}, 1.36 \mathrm{~mm}$, and $1.55 \mathrm{~mm}$, respectively. Eccles et al. [5] reported the average mean difference in the liver surface position as $-0.9 \mathrm{~mm},-0.5 \mathrm{~mm}$, and $0.2 \mathrm{~mm}$ in the CC, AP, and LR directions, with standard deviations of $1.5 \mathrm{~mm}$, $1.5 \mathrm{~mm}$, and $1.5 \mathrm{~mm}$, respectively. Dawson et al. [6] reported that the average intra-fractional CC reproducibility of the diaphragm position relative to the vertebral bone for $\mathrm{BH}$ with $\mathrm{ABC}$ is $2.5 \mathrm{~mm}$.
Owing to the differences in organ motion management techniques and setup procedures among the studies, it is difficult to directly compare these data with our data. The intra-BH variation in tumor position is small, even in lung cancer patients using the Abches system [19-21].

\section{Inter-fractional (non-CECT) variation in liver position}

Next, we investigated the inter-fractional variations (non-CECT) in liver position under the expiratory phase $\mathrm{BH}$ with the Abches system using $\mathrm{CBCT}$. The average inter-fractional variation in liver position in the $\mathrm{CC}$ direction under $\mathrm{CBCT}$ was $2.5 \mathrm{~mm}$. Internal rotation of the liver is very unlikely to be detected on CBCT without using a CE agent. Many authors have reported inter-fractional variations in liver position using the $\mathrm{ABC}$ or $\mathrm{Abches}$ system. Lu et al. [9] reported that the mean absolute inter-fractional displacements in the liver in the LR, $\mathrm{AP}$, and $\mathrm{CC}$ directions under $\mathrm{ABC}$ were $1.78 \mathrm{~mm}$, $2.64 \mathrm{~mm}$, and $2.97 \mathrm{~mm}$, respectively. Dawson et al. [6] reported that the average inter-fractional CC, AP, and LR excursion of the hepatic microcoils were $6.6 \mathrm{~mm}, 3.2 \mathrm{~mm}$, and $3.3 \mathrm{~mm}$, respectively. Kawahara et al. [12] reported that the inter-fractional liver position variations in the LR, AP, and CC directions were $\pm 6 \mathrm{~mm}, \pm 8 \mathrm{~mm}$, and $\pm 15 \mathrm{~mm}$, respectively. The large displacement values reported by Kawahara et al. may have resulted from the side effects of the CE agent in the respiratory phase. They reported that the patient was administered the CE agent, which may have caused a side effect because the patient was fasting. Patient's unstable condition may affect the position of the diaphragm. Potential underlying lung function could also affect patient's ability to breath hold.

\section{Inter-fractional (CECT) variation in liver position}

Finally, we investigated the inter-fractional (CECT) variation in liver position under the expiratory phase $\mathrm{BH}$ with the Abches system using CECT images. The inter-fractional variation in liver position with the CECT were significantly higher than those with the non-CECT. A maximum liver position variation of $27.7 \mathrm{~mm}$ was observed in the CC direction, indicating that patient was unable to reproduce breath-holds at the same position. We observed up to $0.3^{\circ}$ (pitch), $2.2^{\circ}$ (roll), and $2.3^{\circ}$ (yaw) 
maximum rotations of the liver for bony anatomy on CECT. Bertholet et al. reported a maximum rotation of $16.9^{\circ}$ (roll) and $24.6^{\circ}$ (yaw) under marker constellation for bony anatomy [23]. Wunderink et al. reported day-to-day marker constellation rotations of up to $7.2^{\circ}$ in the exhale phase and a low correlation between the bony anatomy and marker displacements [24]. Cao et al. showed that 56.0\%, $14.7 \%$, and $1.3 \%$ of treated fractions had rotational errors greater than $1^{\circ}, 2^{\circ}$, and $3^{\circ}$, respectively, in each of the rotational axes [25]. These reports used abdominal compression for respiratory management. In our study, the rotational motion was small on CT and CBCT without using a $\mathrm{CE}$ agent, given that breathing motion mostly induces liver deformation and rotation. Our results indicated increased variation in liver position on CECT compared with non-CECT under expiratory phase $\mathrm{BH}$. Controlling the inter-fractional liver position under expiratory phase $\mathrm{BH}$ using the Abches system was challenging owing to the differences in $\mathrm{BH}$, daily $\mathrm{BH}$, and the daily condition of the patient. Moreover, the patient could have been nervous during the planning CT because they were subjected to $\mathrm{BH}$ with the Abches system for the first time. Pattern of $\mathrm{CE}$ enhancement is highly specific for hepatocellular carcinoma, CT scan timing is preseted prior to scanning. Patient have to match the CT scan timing with auto-voice system on CT. Matching the timing with auto-voice system on CT is difficult for some patients. If the CECT image is used not only for target delineation but also for image guidance, the patient's condition during the CT simulation may be very different from that during the treatment. The variation in the patient's condition should be small between the simulation and treatment sections for accurate radiotherapy. The inter-fractional variations in liver position for non-CECT were on average less than $3 \mathrm{~mm}$ in the CC direction; however, considerable inter-fractional variations in liver position were observed for CECT under expiratory $\mathrm{BH}$. Significant variations in liver position depend on the presence or absence of a CE agent. Based on our data, non-CECT is more reliable for the determination of tumor position with the $\mathrm{BH}$ technique than CECT for liver SBRT.

\section{Deformation of the liver}

$\mathrm{kV}$-CBCT is widely available for compensating for inter-fractional motion and is clinically essen- tial for SBRT patients with liver cancer. However, as the liver may deform during breathing, we believe that non-CECT images should be used for IGRT in cases without transarterial chemoembolization (TACE) or fiducial markers [26]. Organ deformation is mainly due to physiological variations that are difficult to control and may not be reproducible. The heartbeat and the resulting pulsatile blood flow can lead to further deformation of the liver. When using CECT for a treatment plan, liver organ deformation may not be representative of the tumor, and uncertainty exists in the use of standard surrogates for IGRT. Hawkins et al. reported that some patients exhibited more than $5 \mathrm{~mm}$ of deformation in the liver in the CC and LR directions between pCT and CBCT [15]. Further studies should investigate the impact of $\mathrm{CE}$ agents on liver deformation using deformable image registration methods [27-28]. The selection of the treatment planning CT should be carefully considered when applying CT data with a CE agent for IGRT. CECT images for liver SBRT with $\mathrm{BH}$ should only be used for target delineation and not for treatment planning or IGRT. Even if the shape of the liver and location of the lesion remain constant from CT simulation to treatment, target position correction should be considered when the target is near the OAR that is easily deformable, such as the stomach, duodenum, and small bowel.

\section{Limitation}

A limitation of the current study was our limited ability to visualize liver tumors on CT and CBCT without the use of a CE agent. Therefore, we investigated the tumor position using the shape of the liver and surrounding tissues, such as vessels. CBCT with a $\mathrm{CE}$ agent can be used to discriminate the tumor better than a CBCT scan without a CE agent, as reported by Eccles et al. [29]. They also reported a clinically insignificant effect of the $\mathrm{CE}$ agent on delivered doses, given that the majority of the $\mathrm{CE}$ agent would be washed out after CBCT. Similar to their procedure, $\mathrm{CBCT}$ without a $\mathrm{CE}$ agent should be performed to confirm the difference in liver position between IGRT and delivery durations before intravenous CBCT with a CE agent. Studies have shown that the dosimetric effects of the $\mathrm{CE}$ agent on megavoltage photon dosimetry are clinically insignificant [30]. For patients with significant 
variations in liver position, the dose to the GTV is significantly reduced [10]. Cao et al. reported that the simulated role of various degrees showed that the dose-volume coverage significantly decreased when the rotation exceeded $3^{\circ}[25]$. Rotational errors for liver SBRT using the Abches system under expiratory phase $\mathrm{BH}$ are relatively small in magnitude and are not likely to significantly affect GTV coverage. Further studies are needed to consider the dosimetric impact on patients with poor $\mathrm{BH}$ reproducibility.

\section{Conclusions}

Intra- and inter-fractional variations in liver position were relatively small in non-CECT and CBCT. However, significant inter-fractional variation in liver position on CECT was observed under expiratory phase BH. Significant inter-fraction variation in liver position could become inevitable on account of physiological variations resulting from the CE agent. The position of the liver should be carefully considered when applying CECT data to the treatment plan and IGRT.

\section{Conflict of interest}

The authors declare that they have no conflict of interest to disclose.

\section{Funding}

This publication was prepared without any external source of funding

\section{References}

1. Davies SC, Hill AL, Holmes RB, et al. Ultrasound quantitation of respiratory organ motion in the upper abdomen. Br J Radiol. 1994; 67(803): 1096-1102, doi: 10.1259/00071285-67-803-1096, indexed in Pubmed: 7820402.

2. Balter JM, Dawson LA, Kazanjian S, et al. Determination of ventilatory liver movement via radiographic evaluation of diaphragm position. Int J Radiat Oncol Biol Phys. 2001; 51(1): 267-270, doi: 10.1016/s0360-3016(01)01649-2, indexed in Pubmed: 11516877.

3. Keall PJ, Mageras GS, Balter JM, et al. The management of respiratory motion in radiation oncology report of AAPM Task Group 76. Med Phys. 2006; 33(10): 3874-3900, doi: 10.1118/1.2349696, indexed in Pubmed: 17089851.

4. Kimura T, Hirokawa Y, Murakami Y, et al. Reproducibility of organ position using voluntary breath-hold method with spirometer for extracranial stereotactic radiotherapy. Int J Radiat Oncol Biol Phys. 2004; 60(4): 1307-1313, doi: 10.1016/j.ijrobp.2004.07.718, indexed in Pubmed: 15519804.
5. Eccles C, Brock KK, Bissonnette JP, et al. Reproducibility of liver position using active breathing coordinator for liver cancer radiotherapy. Int J Radiat Oncol Biol Phys. 2006; 64(3): 751-759, doi: 10.1016/j.jijobp.2005.05.066, indexed in Pubmed: 16458774.

6. Dawson LA, Brock KK, Kazanjian S, et al. The reproducibility of organ position using active breathing control $(A B C)$ during liver radiotherapy. Int J Radiat Oncol Biol Phys. 2001; 51(5): 1410-1421, doi: 10.1016/s03603016(01)02653-0, indexed in Pubmed: 11728702.

7. Beddar AS, Kainz K, Briere TM, et al. Correlation between internal fiducial tumor motion and external marker motion for liver tumors imaged with 4D-CT. Int J Radiat Oncol Biol Phys. 2007; 67(2): 630-638, doi: 10.1016/j. ijrobp.2006.10.007, indexed in Pubmed: 17236980.

8. Yang J, Cai J, Wang H, et al. Is diaphragm motion a good surrogate for liver tumor motion? Int J Radiat Oncol Biol Phys. 2014; 90(4): 952-958, doi: 10.1016/j. ijrobp.2014.07.028, indexed in Pubmed: 25223297.

9. Lu L, Diaconu C, Djemil T, et al. Intra- and inter-fractional liver and lung tumor motions treated with SBRT under active breathing control. J Appl Clin Med Phys. 2018; 19(1): 39-45, doi: 10.1002/acm2.12220, indexed in Pubmed: 29152835.

10. Lu L, Ouyang Zi, Lin S, et al. Dosimetric assessment of patient-specific breath-hold reproducibility on liver motion for SBRT planning. J Appl Clin Med Phys. 2020; 21(7): 77-83, doi: 10.1002/acm2.12887, indexed in Pubmed: 32337841.

11. Park JC, Park SHo, Kim JH, et al. Liver motion during cone beam computed tomography guided stereotactic body radiation therapy. Med Phys. 2012; 39(10): 6431-6442, doi: 10.1118/1.4754658, indexed in Pubmed: 23039678.

12. Kawahara D, Ozawa $S$, Nakashima $T$, et al. Interfractional diaphragm changes during breath-holding in stereotactic body radiotherapy for liver cancer. Rep Pract Oncol Radiother. 2018; 23(2): 84-90, doi: 10.1016/j.rpor.2018.01.007, indexed in Pubmed: 29463958.

13. Guckenberger M, Sweeney RA, Wilbert J, et al. Imageguided radiotherapy for liver cancer using respiratorycorrelated computed tomography and cone-beam computed tomography. Int J Radiat Oncol Biol Phys. 2008; 71(1): 297-304, doi: 10.1016/j.jjrobp.2008.01.005, indexed in Pubmed: 18406894.

14. Case RB, Sonke JJ, Moseley DJ, et al. Inter- and intrafraction variability in liver position in non-breath-hold stereotactic body radiotherapy. Int J Radiat Oncol Biol Phys. 2009; 75(1): 302-308, doi: 10.1016/j.jijobp.2009.03.058, indexed in Pubmed: 19628342.

15. Hawkins MA, Brock KK, Eccles C, et al. Assessment of residual error in liver position using $\mathrm{kV}$ cone-beam computed tomography for liver cancer high-precision radiation therapy. Int J Radiat Oncol Biol Phys. 2006; 66(2): 610-619, doi: 10.1016/j.ijrobp.2006.03.026, indexed in Pubmed: 16966004.

16. Zhong $\mathrm{R}$, Wang J, Jiang $X$, et al. Hypofraction radiotherapy of liver tumor using cone beam computed tomography guidance combined with active breath control by long breath-holding. Radiother Oncol. 2012; 104(3): 379-385, doi: 10.1016/j.radonc.2011.11.007, indexed in Pubmed: 22260848.

17. Choi GW, Suh Y, Das P, et al. Assessment of setup uncertainty in hypofractionated liver radiation therapy 
with a breath-hold technique using automatic image registration-based image guidance. Radiat Oncol. 2019; 14(1): 154, doi: 10.1186/s13014-019-1361-6, indexed in Pubmed: 31470860.

18. Onishi $\mathrm{H}$, Kawakami $\mathrm{H}$, Marino K, et al. A simple respiratory indicator for irradiation during voluntary breath holding: a one-touch device without electronic materials. Radiology. 2010; 255(3): 917-923, doi: 10.1148/radiol.10090890, indexed in Pubmed: 20501729.

19. Tarohda $\mathrm{Tl}$, Ishiguro M, Hasegawa $\mathrm{K}$, et al. The management of tumor motions in the stereotactic irradiation to lung cancer under the use of Abches to control active breathing. Med Phys. 2011; 38(7): 4141-4146, doi: 10.1118/1.3604151, indexed in Pubmed: 21859015.

20. Miura H, Ozawa S, Nakao M, et al. Evaluation of interbreath-hold lung tumor position reproducibility with vector volume histogram using the breathhold technique. Med Dosim. 2020; 45(3): 252-255, doi: 10.1016/j.meddos.2020.01.003, indexed in Pubmed: 32063423.

21. Fuss M, Shi C, Papanikolaou N. Tomotherapeutic stereotactic body radiation therapy: Techniques and comparison between modalities. Acta Oncol. 2006; 45(7): 953-960, doi: 10.1080/02841860600897942, indexed in Pubmed: 16982563.

22. Kobayashi D, Takahashi O, Ueda T, et al. Risk factors for adverse reactions from contrast agents for computed tomography. BMC Med Inform Decis Mak. 2013; 13: 18, doi: 10.1186/1472-6947-13-18, indexed in Pubmed: 23363607.

23. Bertholet J, Worm E, Høyer M, et al. Cone beam CT-based set-up strategies with and without rotational correction for stereotactic body radiation therapy in the liver. Acta Oncol. 2017; 56(6): 860-866, doi: 10.1080/0284186X.201 7.1288925, indexed in Pubmed: 28464747.
24. Wunderink W, Méndez Romero A, Seppenwoolde Y, et al. Potentials and limitations of guiding liver stereotactic body radiation therapy set-up on liver-implanted fiducial markers. Int J Radiat Oncol Biol Phys. 2010; 77(5): 1573-1583, doi: 10.1016/j.ijrobp.2009.10.040, indexed in Pubmed: 20399034.

25. Cao M, Lasley FD, Das IJ, et al. Evaluation of rotational errors in treatment setup of stereotactic body radiation therapy of liver cancer. Int J Radiat Oncol Biol Phys. 2012; 84(3): e435-e440, doi: 10.1016/j.jjrobp.2012.05.018, indexed in Pubmed: 22727886.

26. Yue J, Sun X, Cai J, et al. Lipiodol: a potential direct surrogate for cone-beam computed tomography image guidance in radiotherapy of liver tumor. Int J Radiat Oncol Biol Phys. 2012; 82(2): 834-841, doi: 10.1016/j. ijrobp.2010.12.050, indexed in Pubmed: 21377291.

27. Xu Q, Hanna G, Grimm J, et al. Quantifying rigid and nonrigid motion of liver tumors during stereotactic body radiation therapy. Int J Radiat Oncol Biol Phys. 2014; 90(1): 94-101, doi: 10.1016/j.ijrobp.2014.05.007, indexed in Pubmed: 25195990.

28. Brock KK, Hawkins M, Eccles $C$, et al. Improving image-guided target localization through deformable registration. Acta Oncol. 2008; 47(7): 12791285, doi: 10.1080/02841860802256491, indexed in Pubmed: 18766475.

29. Eccles CL, Tse RV, Hawkins MA, et al. Intravenous contrastenhanced cone beam computed tomography (IVCBCT) of intrahepatic tumors and vessels. Adv Radiat Oncol. 2016; 1(1): 43-50, doi: 10.1016/j.adro.2016.01.001, indexed in Pubmed: 28740872.

30. Rankine AW, Lanzon PJ, Spry NA. Effect of contrast media on megavoltage photon beam dosimetry. Med Dosim. 2008; 33(3): 169-174, doi: 10.1016/j.meddos.2007.04.007, indexed in Pubmed: 18674680. 